\title{
Pricing options on variance in affine stochastic volatility models
}

\author{
Jan Kallsen* Johannes Muhle-Karbe ${ }^{\dagger} \quad$ Moritz Voß ${ }^{\ddagger}$
}

\begin{abstract}
We consider the pricing of options written on the quadratic variation of a given stock price process. Using the Laplace transform approach, we determine semi-explicit formulas in general affine models allowing for jumps, stochastic volatility and the leverage effect. Moreover, we show that the joint dynamics of the underlying stock and a corresponding variance swap again are of affine form. Finally, we present a numerical example for the Barndorff-Nielsen and Shephard model with leverage [1]. In particular, we study the effect of approximating the quadratic variation with its predictable compensator.
\end{abstract}

Key words: quadratic variation, realized variance, volatility swap, affine process, stochastic volatility, leverage effect, Laplace transform approach

\section{Introduction}

Due to growing trading volume, the valuation of options written on the realized variance

$$
\sum_{n=1}^{N} \log \left(S_{t_{n}} / S_{t_{n-1}}\right)^{2}=\sum_{n=1}^{N}\left(X_{t_{n}}-X_{t_{n-1}}\right)^{2}, \quad 0=t_{0}<\ldots<t_{N}=T
$$

of a stock $S=S_{0} \exp (X)$, has been studied increasingly, cf. [27] and the list of references therein. Most work has focused on the continuous time approximation of $(1.1)$ by the quadratic variation $[X, X]$ of the log-price $X$ since the latter is considerably more tractable from a mathematical point of view. This approach is justified by the fact that the realized variance $(1.1)$ converges to $[X, X]_{T}$ in probability as the mesh size $\sup _{k=1, \ldots, N}\left|t_{k}-t_{k-1}\right|$ tends to zero (see e.g. [17, I.4.47]). [6] and [27] confirm that the approximation works quite

* Mathematisches Seminar, Christian-Albrechts-Universität zu Kiel, Christian-Albrechts-Platz 4, 24098 Kiel, Germany, (e-mail: kallsen@math.uni-kiel.de).

${ }^{\dagger}$ Fakultät für Mathematik, Universität Wien, Nordbergstraße 15, 1090 Wien, Austria, (e-mail: johannes.muhle-karbe@univie.ac.at).

${ }^{\ddagger}$ Laboratoire de Probabilités et Modèles Aléatoires, Université Pierre et Marie Curie, 175, rue du Chevaleret, 75013 Paris (e-mail: moritz.voss@etu.upmc.fr). 
well for daily fixings $t_{n}$. Consequently, we will only deal with the pricing of options written on the quadratic variation $[X, X]$ of some log-price $X$.

One can broadly divide the existing literature into two distinct categories. If the underlying stock price process is modelled as a continuous semimartingale, Carr and Lee [10, 9] as well as Friz and Gatheral [15] propose model-free valuation approaches based on a replicating portfolio of European options. In the presence of jumps or correlation between stock returns and volatility, one instead has to specify a parametric model. Previous work in this area includes [8], considering Lévy processes, [5], dealing with the model of BarndorffNielsen and Shephard [1, henceforth BNS] without leverage, as well as [6] and [27], which use the Bates model [2] resp. the Heston model augmented by specific jumps in stock and volatility. In related work, [25] and [16] price log-contracts resp. options written on the predictable quadratic variation $\langle X, X\rangle$ in time-changed Lévy models with Cox-Ingersoll-Ross type activity process.

In this paper we study the valuation of options written on quadratic variation in the unifying framework of affine stochastic volatility models. More specifically, we suppose that the stochastic volatility $v$ and the log-price $X$ are modelled as a bivariate affine process in the sense of [14]. This class of models encompasses the specific models that have been considered in the context of options on variance so far. Moreover, it includes most other option pricing models that have been proposed in the literature, as e.g. the BNS model with leverage and its generalization to time-changed Lévy models by [7, 12].

We show that the affine structure of the stochastic volatility model $(v, X)$ is passed on to $(v, X,[X, X])$. This in turn allows to compute the corresponding characteristic function by solving some generalized Riccati equations. With the characteristic function at hand, we can proceed to price options on quadratic variation using Laplace resp. Fourier transform methods as proposed by [11, 21]. Moreover, we valuate options written on the predictable compensator $\langle X, X\rangle$ of $[X, X]$, which differs from the quadratic variation in the presence of jumps.

Afterwards, we also determine the joint dynamics of the market spanned by the stock and a variance swap. Combined with the stochastic volatility process, this market again turns out to be an affine process. This opens the door to variance-optimal hedging of options on quadratic variation in models with jumps, which is subject to current research.

Finally, we present a numerical example for the BNS model with leverage. Here, we investigate to what extent the predictable quadratic variation $\langle X, X\rangle$ can be used as a proxy for the quadratic variation $[X, X]$ in the context of option pricing.

This paper is organized as follows. We start by recalling the notion of semimartingale characteristics. In Section 3 we introduce our general affine stochastic volatility model. Subsequently, we study the quadratic variation process $[X, X]$ of the log-price $X$. In Section 5 we likewise investigate the properties of the predictable quadratic variation $\langle X, X\rangle$ in affine models. We then turn to the pricing of options written on quadratic variation before studying the market consisting of the stock and a variance swap in Section 7. We conclude with a numerical example. 
As for stochastic background and terminology, we refer to the monograph of Jacod and Shiryaev [17]. We write $\mathbb{C}_{-}$for the complex numbers with non-positive real part. For an $\mathbb{R}^{d}$-valued Lévy process $Y$ with Lévy-Khintchine triplet $(\beta, \gamma, \kappa)$, we denote by

$$
\psi^{Y}(u)=u^{\top} \beta+\frac{1}{2} u^{\top} \gamma u+\int\left(e^{u^{\top} x}-1-\left(h\left(x_{1}\right), \ldots, h\left(x_{d}\right)\right)^{\top} u\right) \kappa(d x)
$$

the corresponding Lévy exponent, i.e. the continuous function $\psi^{Y}: i \mathbb{R}^{d} \rightarrow \mathbb{C}$ such that $E\left(e^{u Y_{t}}\right)=\exp \left(t \psi^{Y}(u)\right)$. Here, $h$ denotes a truncation function on $\mathbb{R}$, as e.g. $h(x)=$ $x 1_{\{|x| \leq 1\}}$. Likewise, for a process $Y$ which is affine relative to Lévy-Khintchine triplets $\left(\beta_{i}, \gamma_{i}, \kappa_{i}\right), i=0, \ldots, d$, we write $\psi_{j}^{Y}$ for the Lévy exponent corresponding to the $j$-th triplet $\left(\beta_{j}, \gamma_{j}, \kappa_{j}\right)$.

\section{Differential characteristics}

This paper uses semimartingale characteristics to describe the behavior of stochastic processes. For the convenience of the reader we recall a few of the basic notions here. For a more thorough introduction, we refer to [18] and [17].

To any $\mathbb{R}^{d}$-valued semimartingale $Y$ there is associated a triplet $(B, C, \nu)$ of characteristics, where $B$ resp. $C$ denote $\mathbb{R}^{d}$ - resp. $\mathbb{R}^{d \times d}$-valued predictable processes and $\nu$ a predictable random measure on $\mathbb{R}_{+} \times \mathbb{R}^{d}$. The first characteristic $B$ depends on a truncation function as e.g. $h(x)=x 1_{\{|x| \leq 1\}}$, which is chosen a priori. The characteristics of most processes in applications are absolutely continuous in time, i.e. they can be written as

$$
B_{t}=\int_{0}^{t} b_{s} d s, \quad C_{t}=\int_{0}^{t} c_{s} d s, \quad \nu([0, t] \times G)=\int_{0}^{t} K_{s}(G) d s \quad \forall G \in \mathscr{B}^{d},
$$

with predictable processes $b, c$ and a transition kernel $K$ from $\left(\Omega \times \mathbb{R}_{+}, \mathscr{P}\right)$ into $\left(\mathbb{R}^{d}, \mathscr{B}^{d}\right)$. In this case we call $(b, c, K)$ the differential or local characteristics of $Y$. We implicitly assume that $(b, c, K)$ is a good version in the sense that the values of $c$ are non-negative symmetric matrices, $K_{s}(\{0\})=0$ and $\int\left(1 \wedge|x|^{2}\right) K_{s}(d x)<\infty$.

From an intuitive viewpoint one can interpret differential characteristics as a local LévyKhintchine triplet. Very loosely speaking, a semimartingale with differential characteristics $(b, c, K)$ resembles locally after $t$ a Lévy process with triplet $(b, c, K)(\omega, t)$, i.e. with drift rate $b$, diffusion matrix $c$, and jump measure $K$. Indeed, $Y$ is a Lévy process if and only if the differential characteristics are deterministic and constant, cf. [17, II.4.19].

Affine processes generalize Lévy processes by moving from constant differential characteristics $(b, c, K)$ to affine functions of $Y_{t-}$ in the following sense:

$$
\begin{gathered}
b_{t}(\omega)=\beta_{0}+\sum_{i=1}^{d} Y_{t-}^{i}(\omega) \beta_{i}, \quad c_{t}(\omega)=\gamma_{0}+\sum_{i=1}^{d} Y_{t-}^{i}(\omega) \gamma_{i}, \\
\kappa_{t}(\omega, G)=\kappa_{0}(G)+\sum_{i=1}^{d} Y_{t-}^{i}(\omega) \kappa_{i}(G) \quad \forall G \in \mathscr{B}^{d} .
\end{gathered}
$$


Here, $\left(\beta_{i}, \gamma_{i}, \kappa_{i}\right)$ denote $d+1$ given Lévy-Khintchine triplets on $\mathbb{R}^{d}$. In order to ensure the existence of a semimartingale $Y$ with the specified characteristics, these triplets cannot be chosen arbitrarily. Much rather they have to satisfy certain admissibility conditions to ensure e.g. that the matrix $c$ remains non-negative definite. This issue has been investigated in full generality in [14], which shows that given admissibility of the respective triplets, the characteristic function of the respective affine process can be computed by solving some generalized Riccati equations. A reformulation in terms of semimartingale calculus can be found in [18]. For our stochastic volatility model in Section 3, admissibility is ensured by the conditions required there.

\section{Affine stochastic volatility models}

Our mathematical framework for a frictionless market model is as follows. Fix a terminal time $T>0$ and a filtered probability space $\left(\Omega, \mathscr{F},\left(\mathscr{F}_{t}\right)_{t \in[0, T]}, P\right)$. For simplicity, we assume zero interest rates on a risk-free asset $S^{0}$ with price $S_{t}^{0}=1$ for all $t \in[0, T]$. Furthermore, we suppose that the stochastic volatility $v$ and the logarithm of a stock price $S=S_{0} \exp (X)$ are modelled as a bivariate affine process. This means that the differential characteristics $(b, c, K)$ of the $\mathbb{R}_{+} \times \mathbb{R}$-valued process $(v, X)$ relative to some truncation function $\left(x_{1}, x_{2}\right) \mapsto\left(h\left(x_{1}\right), h\left(x_{2}\right)\right)$ on $\mathbb{R}^{2}$ are of the form

$$
\begin{gathered}
b=\left(\begin{array}{c}
\beta_{0}^{1}+\beta_{1}^{1} v_{-} \\
\beta_{0}^{2}+\beta_{1}^{2} v_{-}
\end{array}\right), \quad c=\left(\begin{array}{cc}
\gamma_{1}^{11} v_{-} & \gamma_{1}^{12} v_{-} \\
\gamma_{1}^{12} v_{-} & \gamma_{0}^{22}+\gamma_{1}^{22} v_{-}
\end{array}\right), \\
K(G)=\kappa_{0}(G)+\kappa_{1}(G) v_{-}, \quad \forall G \in \mathscr{B}^{2} .
\end{gathered}
$$

Here, $\left(\beta_{i}, \gamma_{i}, \kappa_{i}\right), i=0,1$ are given Lévy-Khintchine triplets on $\mathbb{R}^{2}$ which are admissible in the sense that the Lévy measures $\kappa_{0}, \kappa_{1}$ are supported on $\mathbb{R}_{+} \times \mathbb{R}$ and $\beta_{0}^{1}-\int h\left(x_{1}\right) \kappa_{0}(d x)$ is well-defined and positive. Moreover, we assume that $\int_{\left\{x_{1}>1\right\}} x_{1} \kappa_{1}(d x)<\infty$, which ensures that $(v, X)$ does not explode in finite time and hence is a semimartingale in the usual sense (cf. [14, Lemma 9.2, Theorem 2.12]). Finally, we suppose without loss of generality that $X_{0}$ is normalized to zero.

Example 3.1 This class of models includes many specifications that have been proposed in the option pricing literature, as e.g.

1. Lévy processes $X$ with Lévy Khintchine triplet $(b, c, K)$, in which case $\left(\beta_{1}, \gamma_{1}, \kappa_{1}\right)=$ 0 and

$$
\beta_{0}=\left(\begin{array}{l}
0 \\
b
\end{array}\right), \quad \gamma_{0}=\left(\begin{array}{ll}
0 & 0 \\
0 & c
\end{array}\right), \quad \kappa_{0}(G)=\int 1_{G}(0, x) K(d x) \quad \forall G \in \mathscr{B}^{2} .
$$

2. CIR-time-change models of the form

$$
\begin{aligned}
X_{t} & =L_{\int_{0}^{t} v_{s} d s}+\varrho\left(v_{t}-v_{0}\right)+\mu t \\
d v_{t} & =\left(\eta-\lambda v_{t}\right) d t+\sigma \sqrt{v_{t}} d Z_{t}
\end{aligned}
$$


corresponding to

$$
\begin{gathered}
\left.\left(\beta_{0}, \gamma_{0}, \kappa_{0}\right)=\left(\begin{array}{c}
\eta \\
\mu+\varrho \eta
\end{array}\right), 0,0\right) \\
\beta_{1}=\left(\begin{array}{c}
-\lambda \\
b^{L}-\varrho \lambda
\end{array}\right), \quad \gamma_{1}=\left(\begin{array}{cc}
\sigma^{2} & \sigma^{2} \varrho \\
\sigma^{2} \varrho & \sigma^{2} \varrho^{2}+c^{L}
\end{array}\right), \quad \kappa_{1}(G)=\int 1_{G}(0, x) K^{L}(d x),
\end{gathered}
$$

for all $G \in \mathscr{B}^{2}$. Here, $\eta \geq 0, \mu, \varrho, \lambda, \sigma$ are constants, $L$ denotes a Lévy process with triplet $\left(b^{L}, c^{L}, K^{L}\right)$ and $Z$ an independent Wiener process. Note that we recover the dynamics of the Heston model, if $L$ is chosen to be a Brownian motion with drift (cf. [18]).

3. OU-time-change models of the form

$$
\begin{aligned}
& X_{t}=L_{\int_{0}^{t} v_{s} d s}+\varrho Z_{t}+\mu t \\
& d v_{t}=-\lambda v_{t-} d t+d Z_{t}
\end{aligned}
$$

which correspond to

$$
\begin{gathered}
\beta_{0}=\left(\begin{array}{c}
b^{Z} \\
\mu+\varrho b^{Z}
\end{array}\right), \quad \gamma_{0}=0, \quad \kappa_{0}(G)=\int 1_{G}(z, \varrho z) K^{Z}(d z), \\
\beta_{1}=\left(\begin{array}{c}
-\lambda \\
b^{L}
\end{array}\right), \quad \gamma_{1}=\left(\begin{array}{cc}
0 & 0 \\
0 & c^{L}
\end{array}\right), \quad \kappa_{1}(G)=\int 1_{G}(0, x) K^{L}(d x),
\end{gathered}
$$

for all $G \in \mathscr{B}^{2}$. Here, $\mu, \varrho, \lambda$ are constants and $L$ resp. $Z$ denote a Lévy process with triplet $\left(b^{L}, c^{L}, K^{L}\right)$ resp. an independent subordinator with triplet $\left(b^{Z}, 0, K^{Z}\right)$. Observe that we obtain the dynamics of the BNS model if $L$ is chosen to be a Brownian motion with drift, cf. [18].

\section{Quadratic variation}

In this section, we characterize the stochastic volatility $v$, the log-stock price $X$ and the corresponding quadratic variation $[X, X]$ as a trivariate affine process. This in turn leads quickly to the characteristic function and the conditional expectation of $[X, X]$.

Lemma $4.1(v, X,[X, X])$ is affine w.r.t. the triplets $\left(\widehat{\beta}_{i}, \widehat{\gamma}_{i}, \widehat{\kappa}_{i}\right), i=0,1$ given by

$$
\begin{aligned}
& \widehat{\beta}_{0}=\left(\begin{array}{c}
\beta_{0}^{1} \\
\beta_{0}^{2} \\
\gamma_{0}^{22}
\end{array}\right), \quad \widehat{\gamma}_{0}=\left(\begin{array}{ccc}
0 & 0 & 0 \\
0 & \gamma_{0}^{22} & 0 \\
0 & 0 & 0
\end{array}\right), \quad \widehat{\kappa}_{0}(G)=\int 1_{G}\left(x_{1}, x_{2}, x_{2}^{2}\right) \kappa_{0}(d x) \quad \forall G \in \mathscr{B}^{3}, \\
& \widehat{\beta}_{1}=\left(\begin{array}{c}
\beta_{1}^{1} \\
\beta_{1}^{2} \\
\gamma_{1}^{22}
\end{array}\right), \quad \widehat{\gamma}_{1}=\left(\begin{array}{ccc}
\gamma_{1}^{11} & \gamma_{1}^{12} & 0 \\
\gamma_{1}^{12} & \gamma_{1}^{22} & 0 \\
0 & 0 & 0
\end{array}\right), \quad \widehat{\kappa}_{1}(G)=\int 1_{G}\left(x_{1}, x_{2}, x_{2}^{2}\right) \kappa_{1}(d x) \quad \forall G \in \mathscr{B}^{3},
\end{aligned}
$$

relative to the truncation function $\left(x_{1}, x_{2}, x_{3}\right) \mapsto\left(h\left(x_{1}\right), h\left(x_{2}\right), 0\right)$ on $\mathbb{R}^{3}$. 
Proof. By definition, we have $[X, X]=X^{2}-X_{0}^{2}-2 X_{-} \cdot X$. The joint characteristics of $(v, X,[X, X])$ can therefore easily be derived using [18, Propositions 2 and 3].

Since the process $(v, X,[X, X])$ is affine, its characteristic function can be determined by solving some generalized Riccati equations. To simplify notation and since it suffices for our purposes here, we only compute the characteristic function of $[X, X]$ and leave the analogous derivation of its counterpart for $(v, X,[X, X])$ to the interested reader.

Lemma 4.2 For $u \in \mathbb{C}_{-}$, we have

$$
E\left(e^{u[X, X]_{T}} \mid \mathscr{F}_{t}\right)=\exp \left(\Psi_{0}(T-t, u)+\Psi_{1}(T-t, u) v_{t}+u[X, X]_{t}\right),
$$

where $\Psi_{1}(\cdot, u)$ is the unique solution to the initial value problem

$$
\begin{aligned}
\frac{\partial}{\partial t} \Psi_{1}(t, u)= & \frac{\gamma_{1}^{11}}{2} \Psi_{1}^{2}(t, u)+\beta_{1}^{1} \Psi_{1}(t, u)+\gamma_{1}^{22} u \\
& +\int\left(e^{\Psi_{1}(t, u) x_{1}+u x_{2}^{2}}-1-\Psi_{1}(t, u) h\left(x_{1}\right)\right) \kappa_{1}(d x), \\
\Psi_{1}(0, u)= & 0,
\end{aligned}
$$

and

$\Psi_{0}(t, u)=\int_{0}^{t}\left(\beta_{0}^{1} \Psi_{1}(s, u)+\gamma_{0}^{22} u+\int\left(e^{\Psi_{1}(s, u) x_{1}+u x_{2}^{2}}-1-\Psi_{1}(s, u) h\left(x_{1}\right)\right) \kappa_{0}(d x)\right) d s$.

Proof. For $u \in i \mathbb{R}$, the assertion follows from [18, Theorem 3.1]. The extension to $u \in \mathbb{C}_{-}$ is a consequence of [14, Proposition 6.4].

Example 4.3 1. For a Lévy process $X$ with triplet $(b, c, K)$, we have $\Psi_{1}(t, u)=0$ and

$$
\Psi_{0}(t, u)=t\left(c u+\int\left(e^{u x^{2}}-1\right) K(d x)\right) .
$$

This recovers the formula obtained in [8], where the integral w.r.t. the Lévy measure is computed in closed form for the CGMY Lévy process (cf. e.g. [24]). Consequently, the characteristic function of $[X, X]_{T}$ is known explicitly in this case.

2. For CIR-time-change models, $\Psi_{1}$ is given as the solution to the classical Riccati ODE

$$
\frac{\partial}{\partial t} \Psi_{1}(t, u)=\frac{\sigma^{2}}{2} \Psi_{1}^{2}(t, u)-\lambda \Psi_{1}(t, u)+\left(\left(\sigma^{2} \varrho^{2}+c^{L}\right) u+\int\left(e^{u x^{2}}-1\right) K^{L}(d x)\right)
$$

with initial condition $\Psi_{1}(0, u)=0$ and $\Psi_{0}(t, u)=\eta \int_{0}^{t} \Psi_{1}(s, u) d s$. Disregarding the trivial cases where $\lambda=0, \sigma=0$ or $L$ is deterministic, [13, Lemma A.1] and straightforward calculations show

$$
\begin{aligned}
\Psi_{1}(t, u) & =\frac{2 g(u)\left(e^{f(u) t}-1\right)}{f(u)-\lambda+e^{f(u) t}(f(u)+\lambda)} \\
\Psi_{0}(t, u) & =\frac{2 \eta}{\sigma^{2}} \log \left(\frac{2 f(u) e^{t(f(u)+\lambda) / 2}}{f(u)-\lambda+e^{f(u) t}(f(u)+\lambda)}\right) .
\end{aligned}
$$


Here, $\log$ denotes the distinguished logarithm in the sense of [23, Lemma 7.6] and $g(u):=\left(\sigma^{2} \varrho^{2}+c^{L}\right) u+\int\left(e^{u x^{2}}-1\right) K^{L}(d x), f(u):=\sqrt{\lambda^{2}-2 \sigma^{2} g(u)}$. The squareroot represents the principal branch with branch cut along the negative real line. Note that this extends the representations from the proof of [19, Proposition 6.2.5] to $\mathbb{C}_{-}$. If $L$ is chosen to be a Brownian motion with drift (i.e. in the Heston model) or a CGMY Lévy process, all expressions can be evaluated in closed form.

3. For OU-time-change models the situation is more involved because of simultaneous jumps of $v$ and $[X, X]$ for $\varrho \neq 0$. We obtain

$$
\Psi_{1}(t, u)=\frac{1-e^{-\lambda t}}{\lambda}\left(c^{L} u+\int\left(e^{u x^{2}}-1\right) K^{L}(d x)\right),
$$

as the solution to a linear ODE and

$$
\Psi_{0}(t, u)=\int_{0}^{t}\left(b^{Z} \Psi_{1}(s, u)+\int\left(e^{\Psi_{1}(s, u) z+u \varrho^{2} z^{2}}-1-\Psi_{1}(s, u) h(z)\right) K^{Z}(d z)\right) d s .
$$

Hence, $\Psi_{1}$ is known in closed form if $L$ is a Brownian motion with drift (i.e. in the BNS model) or a CGMY Lévy process. Evaluation of the function $\Psi_{0}$, on the other hand, involves one numerical integration even in the BNS model with leverage. Without leverage, i.e. for $\varrho=0$, we obtain $\Psi_{0}(t, u)=\int_{0}^{t} \psi^{Z}\left(\Psi_{1}(s, u)\right) d s$. This integral can be computed in closed form if $v$ is chosen to be e.g. a Gamma- or IG-OU process (see e.g. [20] for more details).

By differentiating the characteristic function, we can compute expectations of $[X, X]_{T}$.

Lemma 4.4 If $\int_{0}^{t} E\left(v_{s}\right) d s<\infty$ and $\int x_{2}^{2} \kappa_{i}(d x)<\infty$ for $i=0,1$, then $[X, X]_{T}$ is integrable and

$$
E\left([X, X]_{T} \mid \mathscr{F}_{t}\right)=\Phi_{0}(t)+\Phi_{1}(t) v_{t}+[X, X]_{t}
$$

where

$$
\Phi_{i}(t):=\left.\frac{\partial}{\partial u} \Psi_{i}(T-t, u)\right|_{u=0}, \quad i=0,1
$$

Proof. Under the stated assumptions, Lemma 4.1] and [17, II.2.29a, II.2.38] imply that the process $[X, X]$ is a special semimartingale with canonical decomposition

$$
[X, X]_{T}=x^{2} *\left(\mu^{X}-\nu^{X}\right)_{T}+\left(\gamma_{0}^{22}+\int x_{2}^{2} \kappa_{0}(d x)\right) T+\left(\gamma_{1}^{22}+\int x_{2}^{2} \kappa_{1}(d x)\right) \int_{0}^{T} v_{s} d s .
$$

Here, $\mu^{X}$ and $\nu^{X}$ denote the random measure of jumps of $X$ and its compensator (cf. [17] for more details). Since

$$
E\left(\left|x^{2} *\left(\mu^{X}-\nu^{X}\right)_{T}\right|\right) \leq 2 E\left(x^{2} * \nu_{T}^{X}\right)=2 T \int x_{2}^{2} \kappa_{0}(d x)+\int x_{2}^{2} \kappa_{1}(d x) \int_{0}^{T} E\left(v_{s}\right) d s
$$


by [17, II.1.8] and Fubini's theorem, $[X, X]_{T}$ is integrable. By exchanging integration and differentiation as in the proof of [4, Satz 25.2], the second assertion follows.

From the representation in Lemma 4.4, one can infer that $\left(v_{t}, X_{t}, E\left([X, X]_{T} \mid \mathscr{F}_{t}\right)\right)_{t \in[0, T]}$ is again an affine process, albeit with time-dependent triplets. This is expounded on in Proposition 7.1 below.

Example 4.5 In our set of concrete specifications, Lemma 4.4 leads to the following results by applying [3, Lemma 16.2] to exchange the order of integration and differentiation.

1. Let $X$ be a Lévy process with triplet $(b, c, K)$ and $\int x^{2} K(d x)<\infty$. Then Lemma 4.4 is applicable with $\Phi_{1}(t)=0$ and

$$
\Phi_{0}(t)=(T-t)\left(c+\int x^{2} K(d x)\right) .
$$

2. For CIR-time-change models satisfying $\int x^{2} K^{L}(d x)<\infty$, we obtain

$$
\begin{aligned}
& \Phi_{1}(t)=\frac{1-e^{-\lambda(T-t)}}{\lambda}\left(\sigma^{2} \varrho^{2}+c^{L}+\int x^{2} K^{L}(d x)\right) \\
& \Phi_{0}(t)=\frac{e^{-\lambda(T-t)}-1+\lambda(T-t)}{\lambda}\left(\sigma^{2} \varrho^{2}+c^{L}+\int x^{2} K^{L}(d x)\right) \frac{\eta}{\lambda} .
\end{aligned}
$$

3. Finally, for OU-time-change models with $\int z^{2} K^{Z}(d z)<\infty$ and $\int x^{2} K^{L}(d x)<\infty$,

$$
\begin{aligned}
\Phi_{1}(t)= & \frac{1-e^{-\lambda(T-t)}}{\lambda}\left(c^{L}+\int x^{2} K^{L}(d x)\right), \\
\Phi_{0}(t)= & \frac{e^{-\lambda(T-t)}-1+\lambda(T-t)}{\lambda^{2}}\left(c^{L}+\int x^{2} K^{L}(d x)\right)\left(b^{Z}+\int(z-h(z)) K^{Z}(d z)\right) \\
& +(T-t) \varrho^{2} \int z^{2} K^{Z}(d z) .
\end{aligned}
$$

\section{Predictable quadratic variation}

For continuous stochastic processes $X$, the quadratic variation $[X, X]$ and its predictable compensator $\langle X, X\rangle$ coincide. For processes with jumps, the two notions differ, even though they have the same expected value under the assumptions of Lemma 4.4 (cf. [17, I.4.50, I.4.2]). For affine models as in Section 3 above, we have the following

Lemma 5.1 Suppose $\int x_{2}^{2} \kappa_{i}(d x)<\infty$ for $i=0,1$. Then $\langle X, X\rangle$ is well-defined and given by

$$
\langle X, X\rangle_{t}=\left(\gamma_{0}^{22}+\int x_{2}^{2} \kappa_{0}(d x)\right) t+\left(\gamma_{1}^{22}+\int x_{2}^{2} \kappa_{1}(d x)\right) \int_{0}^{t} v_{s} d s
$$


Moreover, the conditional characteristic function of $\langle X, X\rangle_{T}$ is given by

$$
E\left(e^{u\langle X, X\rangle_{T}} \mid \mathscr{F}_{t}\right)=\exp \left(\Upsilon_{0}(T-t, u)+\Upsilon_{1}(T-t, u) v_{t}+u\langle X, X\rangle_{t}\right), \quad u \in \mathbb{C}_{-},
$$

where $\Upsilon_{1}(\cdot, u)$ is the unique solution to the initial value problem

$$
\begin{aligned}
\frac{\partial}{\partial t} \Upsilon_{1}(t, u) & =\psi_{1}^{v}\left(\Upsilon_{1}(t, u)\right)+\left(\gamma_{1}^{22}+\int x_{2}^{2} \kappa_{1}(d x)\right) u, \\
\Upsilon_{1}(0, u) & =0,
\end{aligned}
$$

and

$$
\Upsilon_{0}(t, u)=\int_{0}^{t} \psi_{0}^{v}\left(\Upsilon_{1}(s, u)\right) d s+\left(\gamma_{0}^{22}+\int x_{2}^{2} \kappa_{0}(d x)\right) u t
$$

PROOF. The first part of the assertion follows from [17, I.4.52 and II.2.6]. As for the second, notice that an application of [18, Proposition 2] allows to compute the joint differential characteristics of $(v,\langle X, X\rangle)$. Since this process turns out to be affine, [18, Theorem 3.1] yields that the characteristic function of $\langle X, X\rangle$ is of the proposed form.

Notice that $\Upsilon_{0}, \Upsilon_{1}$ coincide with $\Psi_{0}, \Psi_{1}$ for continuous $v$ and $X$, i.e. for $\kappa_{0}=\kappa_{1}=0$. This reflects that $[X, X]=\langle X, X\rangle$ in this case. In the presence of jumps, $\Upsilon_{0}, \Upsilon_{1}$ represent a kind of first-order approximation of $\Psi_{0}, \Psi_{1}$ for small jumps, since they can be obtained from $\Psi_{0}, \Psi_{1}$ by some suitable first-order Taylor expansions of the integrands of the Lévy measures $\kappa_{0}, \kappa_{1}$. Whether this approximation works well or leads to a significant error depends on the specific model and its parameters. A numerical example is presented in Section 8.

Example 5.2 The generalized Riccati ODEs for the characteristic function of $\langle X, X\rangle$ have been thoroughly studied in the context of interest rate theory. In particular, we have

1. $\Upsilon_{1}(t, u)=0$ and $\Upsilon_{0}(t, u)=\left(c+\int x^{2} K(d x)\right) u t$, if $X$ is a Lévy process with triplet $(b, c, K)$ satisfying $\int x^{2} K(d x)<\infty$.

2. For CIR time-change models with $\int x^{2} K^{L}(d x)<\infty$, [13, Lemma A.1] yields

$$
\begin{aligned}
& \Upsilon_{1}(t, u)=\frac{2 q(u)\left(e^{p(u) t}-1\right)}{p(u)-\lambda+e^{p(u) t}(p(u)+\lambda)}, \\
& \Upsilon_{0}(t, u)=\frac{2 \eta}{\sigma^{2}} \log \left(\frac{2 p(u) e^{t(p(u)+\lambda) / 2}}{p(u)-\lambda+e^{p(u) t}(p(u)+\lambda)}\right),
\end{aligned}
$$

for $q(u)=\left(\sigma^{2} \varrho^{2}+c^{L}+\int x^{2} K^{L}(d x)\right) u$ and $p(u)=\sqrt{\lambda^{2}-2 \sigma^{2} q(u)}$.

3. In OU time-change models, the ODE for $\Upsilon_{1}$ is once again linear. We obtain

$$
\begin{aligned}
& \Upsilon_{1}(t, u)=\frac{1-e^{-\lambda t}}{\lambda} u\left(c^{L}+\int x^{2} K^{L}(d x)\right), \\
& \Upsilon_{0}(t, u)=\int_{0}^{t} \psi^{Z}\left(\Upsilon_{1}(s, u)\right) d s+\left(\varrho^{2} \int z^{2} K^{Z}(d z)\right) u t,
\end{aligned}
$$


provided that $\int x^{2} K^{L}(d x)<\infty$ and $\int z^{2} K^{Z}(d z)<\infty$. Note that if the Lévy exponent $\psi^{Z}$ of $Z$ is of a suitable form as e.g. for Gamma-OU or IG-OU processes, $\Upsilon_{0}$ can be evaluated in closed form, (cf. [20]), unlike for quadratic variation.

The characteristic function of the predictable quadratic variation $\langle X, X\rangle$ is considerably easier to compute than its counterpart for the quadratic variation $[X, X]$. However, in the presence of jumps realized variance converges to the latter rather than the former by [17, I.4.47]. We will therefore study the effect of approximating $[X, X]$ with $\langle X, X\rangle$ in the context of option pricing in Section 8 below.

\section{Pricing options on quadratic variation}

We now turn to the valuation of options written on the quadratic variation of the log-price $X$. To this end, we henceforth assume that the dynamics of the process $(v, X)$ are modelled directly under a pricing measure $Q$.

We first consider variance swaps with payoff $[X, X]_{T}-K_{v a r}$ at time $T$. Here, the variance swap rate $K_{v a r}$ is chosen so as to set the initial value $E_{Q}\left([X, X]_{T}-K_{\text {var }}\right)$ of the swap equal to zero. Thus we have the following immediate consequence of Lemma 4.4 .

Corollary 6.1 (Variance swap) Suppose the conditions of Lemma 4.4 hold. Then

$$
K_{v a r}=E_{Q}\left([X, X]_{T}\right)=\Phi_{0}(0)+\Phi_{1}(0) v_{0}
$$

and the price at time tof the variance swap is given by

$$
E_{Q}\left([X, X]_{T}-K_{\text {var }} \mid \mathscr{F}_{t}\right)=\Phi_{0}(t)+\Phi_{1}(t) v_{t}+[X, X]_{t}-K_{\text {var }},
$$

where the functions $\Phi_{0}, \Phi_{1}$ are defined as in Lemma 4.4

Next, we turn to volatility swaps with terminal payoff $\sqrt{[X, X]_{T}}-K_{v o l}$ at time $T$. As above, the volatility swap rate $K_{\text {vol }}$ is chosen so as to set the initial value $E_{Q}\left(\sqrt{[X, X]_{T}}-\right.$ $\left.K_{v o l}\right)$ of the contract equal to zero. Here, the non-linearity introduced by the square root function can be dealt with using the well-known integral representation

$$
\sqrt{x}=\frac{1}{2 \sqrt{\pi}} \int_{0}^{\infty} \frac{1-e^{-u x}}{u^{3 / 2}} d u, \quad x \geq 0,
$$

and Fubini's theorem (cf. e.g. [26]). Combined with Lemma 4.2 this leads to the following

Lemma 6.2 (Volatility swap) Suppose the conditions of Lemma 4.4 hold. Then

$$
K_{v o l}=E_{Q}\left(\sqrt{[X, X]_{T}}\right)=\frac{1}{2 \sqrt{\pi}} \int_{0}^{\infty} \frac{1-\exp \left(\Psi_{0}(T,-u)+\Psi_{1}(T,-u) v_{0}\right)}{u^{3 / 2}} d u,
$$

and the price of the volatility swap at time $t$ satisfies

$$
\begin{aligned}
E_{Q} & \left(\sqrt{[X, X]_{T}}-K_{\text {vol }} \mid \mathscr{F}_{t}\right) \\
& =\frac{1}{2 \sqrt{\pi}} \int_{0}^{\infty} \frac{1-\exp \left(\Psi_{0}(T-t,-u)+\Psi_{1}(T-t,-u) v_{t}-u[X, X]_{t}\right)}{u^{3 / 2}} d u-K_{v o l} .
\end{aligned}
$$


Next, we turn to puts on variance, which can be evaluated in semi-explicit form using the characteristic function of $[X, X]_{T}$, Fubini's theorem and the integral representation

$$
(K-x)^{+}=\frac{1}{2 \pi i} \int_{R-i \infty}^{R+i \infty} \frac{e^{-K z}}{z^{2}} e^{z x} d z=\frac{1}{\pi} \int_{0}^{\infty} \operatorname{Re}\left(\frac{e^{-K(R+i y)}}{(R+i y)^{2}} e^{(R+i y) x}\right) d y,
$$

for $x \geq 0$ and any $R<0$ (cf. e.g. [10, Corollary 7.8] and [22, Theorem 9.2]).

Lemma 6.3 (Variance put) Let $K>0$ and fix $R<0$. Then at time $t \leq T$, the price of a variance put with payoff $\left(K-[X, X]_{T}\right)^{+}$at time $T$ is given by

$$
\begin{aligned}
& E_{Q}\left(\left(K-[X, X]_{T}\right)^{+} \mid \mathscr{F}_{t}\right) \\
& \quad=\frac{1}{\pi} \int_{0}^{\infty} \operatorname{Re}\left(\frac{e^{-K(R+i y)}}{(R+i y)^{2}} e^{\Psi_{0}(T-t, R+i y)+\Psi_{1}(T-t, R+i y) v_{t}+(R+i y)[X, X]_{t}}\right) d y .
\end{aligned}
$$

The corresponding formula for variance calls can immediately be obtained using the put-call parity $(x-K)^{+}=x-K+(K-x)^{+}$combined with Lemmas 4.4 and 6.3 . Furthermore, the price formulas for options written on the predictable quadratic variation are easily obtained by inserting $\Upsilon_{0}$ and $\Upsilon_{1}$ from Lemma 5.1 in place of $\Psi_{0}$ resp. $\Psi_{1}$. Other European options can be dealt with analogously, provided that their payoff admits a suitable integral representation (cf. [10] for more details).

\section{Joint dynamics of stock and variance swap}

We now show that the dynamics of the process $\left(v_{t}, X_{t}, E_{Q}\left([X, X]_{T} \mid \mathscr{F}_{t}\right)\right)_{t \in[0, T]}$ again turn out to be affine. This opens the door to the computation of hedging strategies trading both the underlying stock and a suitable variance swap.

Proposition 7.1 Suppose the prerequisites of Lemma 4.4 hold and the characteristics of $(v, X)$ are given relative to the truncation function $h(x)=x$ on $\mathbb{R}^{2}$.

Then $\left(v_{t}, X_{t}, E_{Q}\left([X, X]_{T}-K_{\text {var }} \mid \mathscr{F}_{t}\right)\right)_{t \in[0, T]}$ is affine in $v$ relative to the time-inhomogenous triplets $\left(\bar{\beta}_{i}(t), \bar{\gamma}_{i}(t), \bar{\kappa}_{i}(t)\right), i=0,1, t \in[0, T]$ given by

$$
\begin{aligned}
& \bar{\beta}_{0}(t)=\left(\begin{array}{c}
\beta_{0}^{1} \\
\beta_{0}^{2} \\
\Phi_{0}^{\prime}(t)+\Phi_{1}(t) \beta_{0}^{1}+\gamma_{0}^{22}+\int x_{2}^{2} \kappa_{0}(d x)
\end{array}\right), \quad \bar{\gamma}_{0}(t)=\left(\begin{array}{ccc}
0 & 0 & 0 \\
0 & \gamma_{0}^{22} & 0 \\
0 & 0 & 0
\end{array}\right), \\
& \bar{\kappa}_{0}(t, G)=\int 1_{G}\left(x_{1}, x_{2}, \Phi_{1}(t) x_{1}+x_{2}^{2}\right) \kappa_{0}(d x) \quad \forall G \in \mathscr{B}^{3}, \\
& \bar{\beta}_{1}(t)=\left(\begin{array}{c}
\beta_{1}^{1} \\
\beta_{1}^{2} \\
\Phi_{1}^{\prime}(t)+\Phi_{1}(t) \beta_{1}^{1}+\gamma_{1}^{22}+\int x_{2}^{2} \kappa_{1}(d x)
\end{array}\right), \\
& \bar{\gamma}_{1}(t)=\left(\begin{array}{ccc}
\gamma_{1}^{11} & \gamma_{1}^{12} & \Phi_{1}(t) \gamma_{1}^{11} \\
\gamma_{1}^{12} & \gamma_{1}^{22} & \Phi_{1}(t) \gamma_{1}^{12} \\
\Phi_{1}(t) \gamma_{1}^{11} & \Phi_{1}(t) \gamma_{1}^{12} & \Phi_{1}^{2}(t) \gamma_{1}^{11}
\end{array}\right) \\
& \bar{\kappa}_{1}(t, G)=\int 1_{G}\left(x_{1}, x_{2}, \Phi_{1}(t) x_{1}+x_{2}^{2}\right) \kappa_{1}(d x) \quad \forall G \in \mathscr{B}^{3} \text {, }
\end{aligned}
$$


with respect to the truncation function $h(x)=x$ on $\mathbb{R}^{3}$.

PROOF. This follows from Corollary 6.1, Lemma 4.1 and Itô's formula for semimartingale characteristics [18, Proposition 3]. Notice that $h(x)=x$ can be used as the truncation function, since $\left(v_{t}, X_{t}, E_{Q}\left([X, X]_{T}-K_{v a r} \mid \mathscr{F}_{t}\right)\right)_{t \in[0, T]}$ is a special semimartingale by [17, II.2.29a].

\section{Numerical illustration}

We now show how to apply our results to the BNS-Gamma-OU model with leverage. This means that the Lévy process $L$ in the OU-time-change model is chosen to be a Brownian motion, which implies that in particular, $c^{L}=1$ and $K^{L}=0$. Moreover, the background driving Lévy process $Z$ is assumed to be a compound Poisson process with exponentially distributed jumps, i.e.

$$
K^{Z}(d z)=1_{(0, \infty)}(z) a b e^{-b z} d z,
$$

for constants $a, b>0$ and $b^{Z}=0$ relative to $h=0$, because $Z$ is constant between jumps. In view of Corollary 6.1 and Example 4.5, it follows that the variance swap rate $K_{v a r}$ is given by

$$
K_{v a r}=\frac{1-e^{-\lambda T}}{\lambda} v_{0}+\left(\frac{e^{-\lambda T}-1+\lambda T}{\lambda^{2}}\right) \frac{a}{b}+\frac{2 a \varrho^{2}}{b^{2}} T .
$$

Moreover, Example 4.3 yields

$$
\Psi_{1}(t, u)=\frac{1-e^{-\lambda t}}{\lambda} u
$$

as well as

$$
\begin{aligned}
\Psi_{0}(t, u) & =a b \int_{0}^{t} \int_{0}^{\infty}\left(e^{\Psi_{1}(s, u) z+u \varrho^{2} z^{2}}-1\right) e^{-b z} d z d s \\
& =\frac{a b}{2 \sqrt{-\varrho^{2} u}} \int_{0}^{t} U\left(\frac{1}{2}, \frac{1}{2}, \frac{\left(b-\Psi_{1}(s, u)\right)^{2}}{-4 \varrho^{2} u}\right) d s-a t,
\end{aligned}
$$

where $U$ denotes the hypergeometric $U$ function. By Lemmas 6.2 and 6.3 , the volatility swap rate $K_{v o l}$ and prices of puts on quadratic variation can therefore be computed by numerically performing a nested integration.

For comparison, we also consider the value of a volatility swap resp. a put option written on the predictable quadratic variation. For the Gamma-OU process, we have $\psi^{Z}(u)=$ $a u /(b-u)$ by e.g. [24, 5.5.1]. Insertion into Lemma 5.1 yields

$$
\begin{aligned}
\Upsilon_{1}(t, u) & =\frac{1-e^{-\lambda t}}{\lambda} u \\
\Upsilon_{0}(t, u) & =\int_{0}^{t} \frac{a \Upsilon_{1}(s, u)}{b-\Upsilon_{1}(s, u)} d s+\left(\varrho^{2} \int z^{2} K^{Z}(d z)\right) u t \\
& =\frac{a}{b \lambda-u}\left(b \log \left(\frac{b-\Upsilon_{1}(t, u)}{b}\right)+u t\right)+\frac{2 a \varrho^{2}}{b^{2}} u t
\end{aligned}
$$


after an elementary integration. Here, log denotes the distinguished logarithm in the sense of [23, Lemma 7.6]. Consequently, the swap rate for the volatility swap and prices of puts on the predictable quadratic variation can be computed by performing a single numerical integration. As for parameters, we use

$$
a=1.4338, \quad b=11.6641, \quad \lambda=0.5783, \quad \varrho=-1.2606, \quad v_{0}=0.0145,
$$

obtained in [24] by calibrating the BNS-Gamma-OU model to a set of 75 call options on the S\&P500. The results are shown in Figures 1 and 2 .

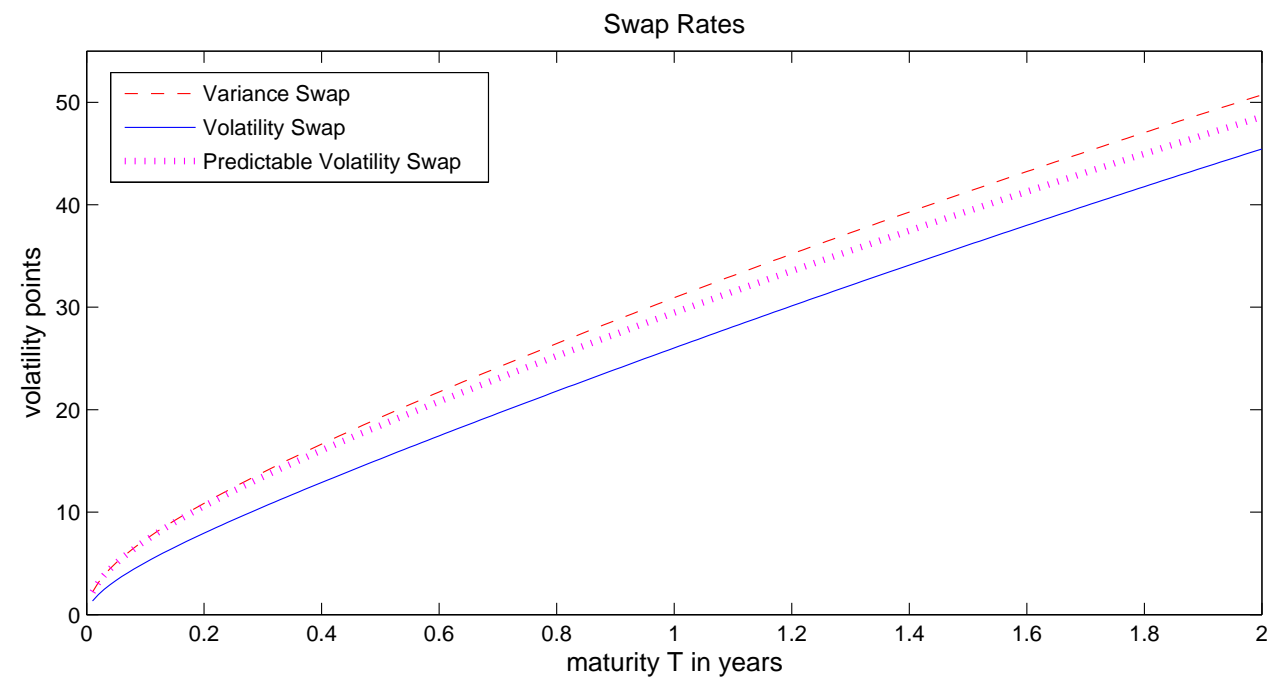

Figure 1: Swap rates in the BNS-Gamma-OU model with leverage.

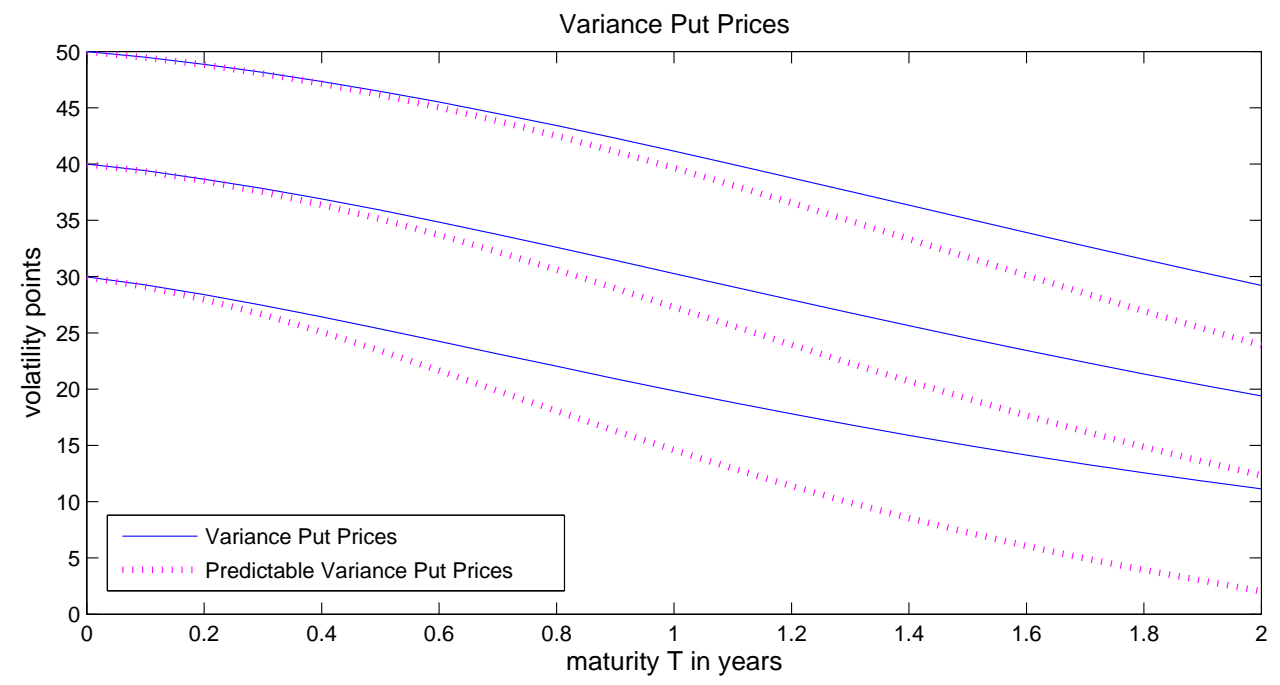

Figure 2: Put prices in the BNS-Gamma-OU model with leverage for strike $K=50$ (top), 40 (middle), 30 (bottom) volatility points. 
The predictable volatility swap rate consistently overestimates the unpredictable one. Similarly, the prices of puts on predictable quadratic variation are always smaller than those for its unpredictable counterpart in our setup. Hence, one should be wary about using $\langle X, X\rangle$ as a proxy for $[X, X]$ to obtain simpler formulas.

\section{References}

[1] O. Barndorff-Nielsen and N. Shephard. Non-Gaussian Ornstein-Uhlenbeck-based models and some of their uses in financial economics. Journal of the Royal Statistical Society, Series B, 63:167-241, 2001.

[2] D. Bates. Jumps and stochastic volatility: Exchange rate processes implicit in deutsche mark options. Review of Financial Studies, 9:69-107, 1996.

[3] H. Bauer. Maß- und Integrationstheorie. de Gruyter, Berlin, second edition, 1992.

[4] H. Bauer. Wahrscheinlichkeitstheorie. de Gruyter, Berlin, fifth edition, 2002.

[5] F. Benth, M. Groth, and R. Kufakunesu. Valuing volatility and variance swaps for a non-Gaussian Ornstein-Uhlenbeck stochastic volatility model. Applied Mathematical Finance, 14:347-363, 2007.

[6] M. Broadie and A. Jain. The effects of jumps and discrete sampling on volatility and variance swaps. International Journal of Theoretical and Applied Finance, 11:761797, 2008.

[7] P. Carr, H. Geman, D. Madan, and M. Yor. Stochastic volatility for Lévy processes. Mathematical Finance, 13:345-382, 2003.

[8] P. Carr, H. Geman, D. Madan, and M. Yor. Pricing options on realized variance. Finance and Stochastics, 9:453-475, 2005.

[9] P. Carr and R. Lee. Realized volatility and variance: Options via swaps. RISK, 20:7683, 2007.

[10] P. Carr and R. Lee. Robust replication of volatility derivatives. Preprint, 2008.

[11] P. Carr and D. Madan. Option valuation using the fast Fourier transform. The Journal of Computational Finance, 2:61-73, 1999.

[12] P. Carr and L. Wu. Time-changed Lévy processes and option pricing. Journal of Financial Economics, 71:113-141, 2004.

[13] A. Černý and J. Kallsen. Mean-variance hedging and optimal investment in Heston's model with correlation. Mathematical Finance, 18:473-492, 2008. 
[14] D. Duffie, D. Filipović, and W. Schachermayer. Affine processes and applications in finance. The Annals of Applied Probability, 13:984-1053, 2003.

[15] P. Friz and J. Gatheral. Valuation of volatility derivatives as an inverse problem. Quantitative Finance, 5:531-542, 2005.

[16] A. Itkin and P. Carr. Pricing swaps and options on quadratic variation under stochastic time change models. Presentation at the 14th Annual CAP Workshop, Columbia University, 2007.

[17] J. Jacod and A. Shiryaev. Limit theorems for stochastic processes. Springer, Berlin, second edition, 2003.

[18] J. Kallsen. A didactic note on affine stochastic volatility models. In Yu. Kabanov, R. Liptser, and J. Stoyanov, editors, From Stochastic Calculus to Mathematical Finance, pages 343-368. Springer, Berlin, 2006.

[19] D. Lamberton and B. Lapeyre. Stochastic Calculus Applied to Finance. Chapman \& Hall, London, 1996.

[20] E. Nicolato and E. Venardos. Option pricing in stochastic volatility models of the Ornstein-Uhlenbeck type. Mathematical Finance, 13:445-466, 2003.

[21] S. Raible. Lévy Processes in Finance: Theory, Numerics, and Empirical Facts. Dissertation Universität Freiburg i. Br., 2000.

[22] W. Rudin. Real and complex analysis. McGraw-Hill, NewYork, third edition, 1987.

[23] K. Sato. Lévy Processes and Infinitely Divisible Distributions. Cambridge University Press, Cambridge, 1999.

[24] W. Schoutens. Lévy Processes in Finance. Wiley, New York, 2003.

[25] W. Schoutens. Moment swaps. Quantitative Finance, 5:525-530, 2005.

[26] K. Schürger. Laplace transforms and suprema of stochastic processes. In Advances in Finance and Stochastics, pages 285-294. Springer, Berlin, 2002.

[27] A. Sepp. Pricing options on realized variance in Heston model with jumps in returns and variance. Journal of Computational Finance, 11:33-70, 2008. 